\title{
IAU COLIOQUIUM 143
}

\section{The Sun as a Variable Star}

Solar and Stellar Irradiance Variations 
How does the variability of the Sun affect the climate on Earth and other conditions in our atmosphere? The 143rd IAU Colloquium, held in Boulder, Colorado, brought together international experts to investigate this connection. This timely volume presents their articles and together these clearly illustrate just how variations in the energy output of the Sun affect the terrestrial climate, radiative environment, and chemistry of the upper atmosphere.

This survey provides a complete and up-to-date review of observations, theoretical interpretations, and empirical and physical models of variations in the energy output of the Sun and solar-type stars. In particular, it shows how this variability is related to magnetic activity on the surface of the star and how long-term modulations are driven by the stellar interior.

Together, these articles clearly demonstrate the link between changes in the radiative output of the Sun and registered climatic anomalies - essential reading for researchers and graduatc students. 
The Sun as a Variable Star 
IAU Astronomical Union

Union Astronomique International

The following Colloquia of the International Astronomical Union are published for the Union by Cambridge University Press.

82. Cepheids. Edited by Barry F. Madore. 052130091 6. 1985

91. History of Oriental Astronomy. Edited by G. Swarup, A. K. Bag and K. S. Shukla. 052134659 2. 1987

92. Physics of Be Stars. Edited by A. Slettebak and T. P. Snow. 0521330785.1987

101. Supernova Remnants and the Interstellar Medium. Edited by R. S. Roger and T. L. Landecker. 052135062 X. 1988

105. The Teaching of Astronomy. Edited by Jay M. Pasachoff and John R. Percy. 0521353319.1990

106. Evolution of Peculiar Red Giant Stars. Edited by Hollis Johnson and Ben Zuckerman. 052136617 8. 1989

111. The Use of Pulsating Stars in Fundamental Problems of Astronomy. Edited by Edward G. Schmidt. 052137023 X. 1989

136. Stellar Photometry - Current Techniques and Future Developments. Edited by C. J. Butler and I. Elliott. 052141866 6. 1993

139. Stellar Pulsation and Pulsating Variable Stars. Edited by James M. Nemec and Jaymie M. Matthews. 052144382 2. 1993

143. The Sun as a Variable Star. Edited by J. M. Pap, C. Fröhlich, H. S. Hudson and S. K. Solanki. 052142006 7. 1994

145. Supernovae and Supernova Remnants. Edited by Richard McCray. 0521460808. 1994

147. The Equation of State in Astrophysics. Edited by Gilles Chabrier and Evry Schatzman. 052147260 1. 1994 


\title{
The Sun as a Variable Star Solar and Stellar Irradiance Variations
}

Proceedings of IAU Colloquium No. 143

held in Boulder, Colorado, USA

June 20-25, 1993

\author{
Edited by \\ J. M. PAP \\ Jet Propulsion Laboratory, \\ California Institute of Technology \\ Pasadena, California, USA \\ C. FRÖHLICH \\ World Radiation Centre, PMOD \\ Davos, Switzerland \\ H. S. HUDSON \\ University of Hawaii, \\ Honolulu, Hawaii, USA \\ S. K. SOLANKI \\ ETH-Institute of Astronomy, \\ Zürich, Switzerland
}


Published by the Press Syndicate of the University of Cambridge

The Pitt Building, Trumpington Street, Cambridge CB2 1RP

40 West 20th Street, New York, NY 10011-4211, USA

10 Stamford Road, Oakleigh, Melbourne 3166, Australia

(C) Cambridge University Press 1994

First published 1994

Printed in Great Britain at the University Press, Cambridge

A catalogue record for this book is available from the British Library

Library of Congress cataloguing in publication data available

ISBN 0521420067 hardback 
Dedicated to the memory of

Ernest Gurtovenko 


\section{Contents}

Participants $\quad$ xiii

Group Photograph xvii

Preface $\quad$ xix

Acknowledgements $\quad \mathrm{xxi}$

Statement from the Scientific Organizing Committee xxiii

\section{Introduction}

A Historical Perspective on Measurements of Solar Irradiance

V. Gaizauskas .......................... 1

Solar Monitoring has a Past and Present: Does it have a Future?

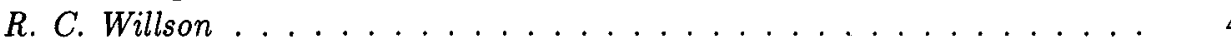

Solar Variability and its Terrestrial Effects

G. L. Withbroe and W. Kalkofen

General Reviews on Observations of Solar and Stellar Irradiance Variability from Space and from the Ground

Irradiance Variations of Stars

G. W. Lockwood . . . . . . . . . . . . . . . . . . . . . . . . .

Irradiance Observations of the Sun

C. Fröhlich . . . . . . . . . . . . . . . . . .

Solar Absolute Reference Spectrum

H. Neckel . . . . . . . . . . . . . . . . . . .

The Solar Spectral Irradiances from X-ray to Radio Wavelengths

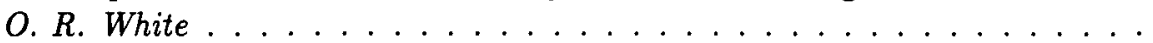

Observational Programs for Solar and Stellar Irradiance Variability

Irradiance Observations of SMM, Spacelab 1, UARS, and ATLAS Experiments $R$. C. Willson

Total Solar Irradiance Observations from the EURECA and ATLAS Experiments D. Crommelynck, V. Domingo, A. Fichot, and R. Lee . . . . . . . . . .

Irradiance Observations in Near-UV, Visible and Infrared Spectral Bands from Measurements Carried out during ATLAS-1 and EURECA-1 Missions

G. Thuillier, M. Herse, D. Labs, P. C. Simon, D. Gillotay, and H. Mandel.

Irradiance Observations from the UARS/SUSIM and ATLAS/SUSIM Experiments G. Brueckner, L. E. Floyd, P. A. Lund, D. K. Prinz, and M. E. VanHoosier

Irradiance Observations from the UARS/SOLSTICE Experiment

G. J. Rottman, T. N. Woods, O. R. White, and J. London .

Middle Ultraviolet Solar Spectral Irradiance Measurements, 1985-1992, from the SBUV/2 and SSBUV Instruments

R. P. Cebula, E. Hilsenrath, and M. T. Deland . . . . . . . . . . . 
The Variable Structure of the X-ray Corona as Viewed by Yohkoh

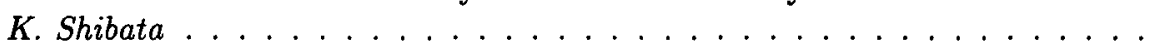

Summary of Future Space Observations at Different Wavelengths

V. Domingo . . . . . . . . . . . . . . . . . . . 101

Photometric Variations of Solar Type Stars

R. R. Radick. . . . . . . . . . . . . . . . . . . 109

Photometric Observations of the Sun

G. A. Chapman . . . . . . . . . . . . . . . . . 117

Brightness Observations of the Sun

J. R. Kuhn . . . . . . . . . . . . . . . . . . . . 130

Observations of Global Solar Magnetic and Velocity Fields

J. T. Hoeksema . . . . . . . . . . . . . . . . .

Variability of Solar and Stellar Irradiance Related to the Network, Active Regions (Sunspots and Plages), and LargeScale Magnetic Structures

The Variability of Stellar Magnetic Flux, Related Surface Structures and Activity S. Saar

Variations of the Coronal Radiation in X-ray Related to Coronal Holes, Active Region Loop Systems, Bright Points

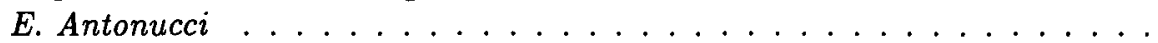

Variations of Coronal Radiations at Optical Wavelengths

R. C. Altrock

Slowly-Varying Microwave Emissions from the Solar Corona

K. F. Tapping and K. L. Harvey . . . . . . . . . . . . . . . .

\section{Empirical Models of Solar Total and Spectral Irradiance Vari- ability}

Spatial Resolution of Solar Total Irradiance Variability: The YOHKOH White-Light Observations

H. S. Hudson

The Sun in Time: Detecting and Modelling Magnetic Inhomogenities on Solar-Type Stars

J. D. Dorren and E. F. Guinan . . . . . . . . . . . . . . . .

Irradiance Models Based on Solar Magnetic Fields

K. L. Harvey . . . . . . . . . . . . . . . . . . . . .

Irradiance Effects of Small-Scale Magnetic Fields on the Sun

S. K. Solanki . . . . . . . . . . . . . . . . .

Estimating Solar Forcing of Climate Change during the Maunder Minimum

J. Lean, A. Skumanich, O. R. White, and D. Rind . . . . . . . . . .

Solar Rotation, Irradiance Changes and Climate

E. Nesme-Ribes, D. Sokoloff, and R. Sadourny. 
Solar and Stellar Oscillations, Irradiance Variations, and their Interpretation

What can we Learn from Oscillation Studies about Irradiance and Radius Changes?

D. Gough . . . . . . . . . . . . . . . . . . . 252

Theoretical Interpretation of Magnetic Activity

E. N. Parker. . . . . . . . . . . . . . . . . . . 264

Theoretical Interpretation of Solar and Stellar Irradiance Variations

H. C. Spruit . . . . . . . . . . . . . . . . . . . . . . . 270

Convection and Irradiance Variations

P. A. Fox and S. Sofia . . . . . . . . . . . . . . . . . 280

The Response of the Earth's Atmosphere to Solar Irradiance Variations and Sun-Climate Connections

Solar Variability Traced by Cosmogenic Isotopes

J. Beer, S. T. Baumgartner, B. Dittrich-Hannen, J. Hauenstein, P. Kubik, C.

Lukasczyk, W. Mende, R. Stellmacher, and M. Suter . . . . . . . . . . . 291

Solar Forcing of Global Climate Change

P. E. Damon and J. L. Jirikowic . . . . . . . . . . . . . . . . . 301

Response of the Middle Atmosphere to Solar Variability - Model Simulations

T. Y. W. Huang and G. Y. Brasseur . . . . . . . . . . . . . 315

A Probable Connection between Solar and Atmospheric Decadal Variability

K. Labitzke and H. Loon . . . . . . . . . . . . . . . . . . . 330

Solar Activity and Global Temperature

E. Friis-Christensen and K. Lassen . . . . . . . . . . . . . . . . . . 339

List of Contributed (Poster) Papers 